The final publication is available at Elsevier via http://dx.doi.org/10.1016/j.jtbi.2017.07.029 ( 2017. This manuscript version is made available under the CC-BY-NC-ND 4.0 license https://creativecommons.org/licenses/by-nc-nd/4.0/

\title{
Competition between injunctive social norms and conservation priorities give rise to complex dynamics in a model of forest growth and opinion dynamics
}

\author{
Ram Prasad Sigdel ${ }^{1}$, Chris T. Bauch ${ }^{1,2}$ \\ ${ }^{1}$ Department of Mathematics and Statistics, University of Guelph \\ ${ }^{2}$ Department of Mathematics and Statistics, University of Waterloo \\ Correspondence: rsigdel@uoguelph.ca
}

\begin{abstract}
Human systems and environmental systems are often treated as existing in isolation from one another, whereas in fact they are often two parts of a single, coupled human-environment system. Developing theoretical models of coupled human-environment systems is a continuing area of research, although relatively few of these models are based on differential equations. Here we develop a simple differential equation coupled human-environment system model of coupled forest growth dynamics and conservationist opinion dynamics in a human population. The model assumes logistic growth and harvesting in the forest. Opinion spread in the human population is based the interplay between conservation values stimulated by forest rarity, and injunctive social norms that tend to support population conformity. We find that injunctive social norms drive the system to the boundaries of phase space, whereas rarity-based conservation priorities drive the system to the interior. The result is complex dynamics including limit cycles and alternative stable states that do not occur if injunctive social norms are absent. We also find that increasing the inherent perceived value of forests is the best way to boost and stabilize forest cover while also boosting conservationist opinion in the population. We conclude that simple models can provide insights and suggest patterns that might be harder to see with complicated, high-dimensional models, and therefore should be pursued more often in research on coupled human-environment systems.
\end{abstract}

Keywords: Imitation dynamics, Socio-ecological system, Differential equation model, Alternative stable states, Regime shift. 


\section{INTRODUCTION}

To meet the growing demand for food, fresh water, timber, fibre and fuel, human beings have been changing ecosystems for substantial gains to their well-being and economic development, but this has also degraded many ecosystem services and increased risk of catastrophic changes in local, regional and global environments (Marten (2001); Francis and Krishnamurthy (2014)). Many natural resources upon which human are depending are either disappeared or are in threat by human action (Millennium Ecosystem Assessment, 2005). According to some scholars, three of nine 'planetary boundaries' which should not be exceeded in order for humanity to operate safely have already been exceeded: biodiversity loss, interruption of nitrogen cycle and climate change (Rockström et al., 2009).

Sustainable land use, including maintaining healthy forest ecosystems, are also one of the nine planetary boundaries, and deforestation is threatening to push us past this boundary as well (Rockström et al., 2009). Due to direct human activities, about 7.5 million hectares of North American forests (or 1\% of total forest area) are disturbed each year (Masek et al., 2011). The Amazon forest has lost at least $17 \%$ of its forest cover, while the Sumatra island of Indonesia lost $85 \%$ of original forests in last five decades due to human activities (WWF, 2013). Intensive ranching is an important culprit in deforestation: it is estimated that $70 \%$ of deforestation in Latin American forest has been caused by cattle ranching operations (Fearnside, 1993).

Forest-grassland mosaics and savanna systems are also under threat. Savanna contain a large proportion of rapidly growing human populations, including much range-land and livestock (Scholes and Archer, 1997). Intensive land use through cultivation and mining activities completely altered $14 \%$ of South African savanna biome (Mogaka, 2001). Similarly, 
the southern Brazilian Campos-Araucaria forest-grassland mosaic is highly diverse (especially the grassland component) but is continuously facing threats in its biodiversity and has lost around 25\% of land in past three decades (Overbeck et al., 2007; Henderson et al., 2016).

A coupled human and environment system (HES or CHES)-also known as coupled human and natural system-is a system in which a human subsystem and an environment subsystem are connected through a coupled feedback loop of mutual influence. Such interactions can apply at local scales, global scales, or across multiple scales (Liu et al., 2007) and can also result in regime shifts between alternative stable states (Lade et al., 2013; Bauch et al., 2016).

For instance, as human activities lead to a decline in natural ecosystems, the decline in natural ecosystems can in turn stimulate human action to conserve endangered ecosystems or attempt to restore badly damaged ecosystems (Heinen and Yonzon, 1994). For instance Sudbury, Canada began replanting trees destroyed by industry-generated acid rain in a massive effort starting in the 1970s, thereby improving both environmental quality and socioeconomic conditions (McCracken, 2013; Anand et al., 2005).

In the past, various mathematical models have explored human-environment interactions in the context of forest dynamics, including models that account for socioeconomic and demographic factors (An et al., 2005); two-person games and their impact on deforestation (Rodrigues et al., 2009); the effect of landowner decision-making on landscape dynamics (Satake and Iwasa, 2006), Markov chain models to study the effect of social learning on 
landowner decisions (Satake et al., 2007); the role of governance in landowner decisionmaking and landscape dynamics (Henderson et al., 2013); and tipping points (Bauch et al., 2016) and regime shifts (Henderson et al., 2016) in coupled forest HESs, among others.

Injunctive social norms-also known as moral norms-are the unwritten but socially acceptable rules and guidelines of a society. Such rules has been established based on their practice and may vary from society to society. The role and influence of injunctive social norms has been accounted for in models of coupled dynamics of vaccinating behaviour and disease dynamics (Oraby et al., 2014), as well as in coupled HES models where it has been studied in the context of forest pest invasion due to human decisions to transport firewood (Barlow et al., 2014); harvesting of a renewable resources, in the form of social ostracism directed toward over-harvesters (Lade et al., 2013); and forest dynamics (Satake et al., 2007).

Previous research in modelling coupled human-environment systems has predominantly relied upon agent-based models (An et al., 2005). In the occasional case where differential equation models have been used, analysis has been primarily numerical, often due to the dimensionality of the system (Henderson et al., 2016), stochasticity (Barlow et al., 2014), nonlinear resource harvesting (Bauch et al., 2016) or use of nonlinear recruitment thresholds (Innes et al., 2013; Henderson et al., 2016). In this paper we develop a simple, differentialequation coupled human-environment system model of a human population making conservation decisions about a forest ecosystem lacking a recruitment threshold. Our objective was to evaluate the influence of injunctive social norms on coupled HES models by comparing models with and without social norms, and to gain general insights by applying the methods of qualitative analysis of ODEs to the system in order to generate more mathematical rigor than has been feasible before in models of coupled HESs. The model also assumes that 
human perception of the conservation priority of the forest system depends on how rare the ecosystem is.

\section{MODEL}

We let $F$ denote the proportion of forest cover in a region, with the remaining land cover $G=1-F$ being other land types include natural grassland, urban spaces, pasture, and other natural or anthropogenic types. Suppose individuals are divided only into two groups, where $x$ is the proportion of forest conservationists and $1-x$ is the proportion of nonconservationists. Here an individual adopts one of the two opinions, either to favour forest conservation $(\mathbb{F})$ or favour other land uses $(\mathbb{G})$. Based on the two strategies, individuals get sources of information and sample one another at the rate $k^{\prime}$. In this case, an individual adopting strategy $\mathbb{F}$ samples other individuals adopting either of the strategies $\mathbb{F}$ or $\mathbb{G}$. Let $\pi(\mathbb{F})$ be the perceived value of forest, and $\pi(\mathbb{G})$ be the perceived value of other land use.

Before switching their strategies, an individual compares utility gain and loss received by adopting the same strategy and may switch strategies if the utility for switching is attractive enough. If $\pi(\mathbb{F})>\pi(\mathbb{G})$ then the one adopting strategy $\mathbb{G}$ switches into strategy $\mathbb{F}$ with probability $\mathrm{p} U_{F}(F)$ (let), where $U_{F}(F)=\pi(\mathbb{F})-\pi(\mathbb{G})>0$ be the net gain in utility by adopting strategy $\mathbb{F}$ than adopting strategy $\mathbb{G}$ and $\mathrm{p}$ is the proportionality constant. Therefore, $(1-x)$

non-conservationists at any given time become conservationists at the rate $(1-x) k^{\prime} x p U_{F}(F)$. Similarly if $U_{G}(F)=\pi(\mathbb{G})-\pi(\mathbb{F})>0$ then $x$ conservationists becomes non-conservationists at the rate $x k^{\prime}(1-x) \alpha p U_{G}(F)$ where, $U_{G}(F)$ is the net gain in utility, by adopting strategy $\mathbb{G}$ than adopting strategy $\mathbb{F}$ and $\alpha$ is the scaling constant. For convenience, we absorb $\alpha$ into $k^{\prime}$ 
Combining above two rates gives:

$$
\begin{aligned}
\frac{d x}{d t} & =\left(k^{\prime} p\right) x(1-x)\left[U_{F}(F)-U_{G}(F)\right] \\
& =k x(1-x)\left[U_{F}(F)-U_{G}(F)\right]
\end{aligned}
$$

where $k=p k^{\prime}$.

From Innes et al. (2013), $U_{F}(F)=r_{0} G-q_{0} F$, where $r_{0}$ and $q_{0}$ control the conservation value (or simply the value/importance) of forest and other land use respectively. In addition to the utility gain $U_{F}(F)$ or $U_{G}(F)$, each member in each social group further feels a uniform pressure $\delta_{0}$ to remains on the same group due to injunctive social norms. Hence an individual adopting a strategy $\mathbb{F}$ experiences the uniform pressure at the rate $\delta_{0} x$ and those adopting $\mathbb{G}$ experience the uniform pressure at the rate $\delta_{0}(1-x)$. Therefore $U_{F}(F)$ and $U_{G}(F)$ in equation (1), can be replaced by $U_{F}(F)+\delta_{0} x$ and $-U_{F}(F)+\delta_{0}(1-x)$ respectively to get,

$$
\begin{aligned}
\frac{d x}{d t} & =k x(1-x)\left[U_{F}(F)+\delta_{0} x+U_{F}(F)-\delta_{0}(1-x)\right] \\
& =k x(1-x)\left[r-m F+\delta_{0}(2 x-1)\right]
\end{aligned}
$$

where $r=2 r_{0}, q=2 q_{0}$ and $m=r+q$. This imitation dynamic has been used in previous models of coupled HESs (Innes et al., 2013; Barlow et al., 2014; Lade et al., 2013) and coupled behavior-disease systems (Oraby et al., 2014).

The corresponding equation for forest cover is a simple logistic population growth model with harvesting

$$
\dot{F}=R F G-h(1-x) F
$$

where $R$ is the natural succession rate of forest from other land and $h$ is the harvesting rate. $h$ can be thought of as a maximal harvesting rate, whereas the realized harvesting rate is 
$h(1-x)$ and can be less than the maximal harvesting rate $h$ due to population support for conservationism. Here, as the new forest depends upon the existing forest and the available land to grow new trees, we consider the new forest is created at the rate proportional to the product of existing forest and other land areas. Also, the harvesting rate is proportional to the product of existing forest cover and proportion of non-conservationists.

Therefore the complete system of equations can be written as

$$
\begin{aligned}
& \dot{x}=\kappa x(1-x)[c-F+\delta(2 x-1)] \\
& \dot{F}=R F(1-F)-h(1-x) F,
\end{aligned}
$$

where $G \equiv 1-F, \kappa \equiv k m, c \equiv \frac{r}{m}$ and $\delta \equiv \frac{\delta_{0}}{m}$. The model variables, parameters, and baseline parameter values appear in Table 1.

TABLE 1. Variables/Parameters, their definition, ranges and baseline values.

\begin{tabular}{|c||c|c|}
\hline Variables & Descriptions & Ranges \\
\hline \hline$x$ & Proportion of conservationist & {$[0,1]$} \\
\hline$F$ & Forest cover & {$[0,1]$} \\
\hline \hline Parameters & Descriptions & Values (Reference/Calibrated) \\
\hline \hline$\kappa$ & Social learning rate (modified) & $0.5 /$ year, Davis et al. (2001) \\
\hline$c$ & Relative conservation value of forest & 0.6 \\
\hline$\delta$ & Strength of injunctive social norms (modified) & 0.01 \\
\hline$R$ & Natural succession rate of forest & $0.01 /$ year \\
\hline$h$ & harvesting efficiency & $0.02 /$ year \\
\hline
\end{tabular}

\section{Results}

We first analyze the model with injunctive social norms included. In the following subsection, we will analyze the special case where injunctive social norms are not included, and then compare the two models.

\subsection{Model with injunctive social norms.}


3.1.1. Model Equilibria. From equation (4), there are two steady states $\left(F^{*}, x^{*}\right)$ of full nonconservationism. One has no forest cover

$$
A_{1}=(0,0)
$$

and the second one has a mix of forest and other land cover

$$
A_{2}=\left(1-\frac{h}{R}, 0\right)
$$

$A_{2}$ is biologically meaningful when the natural succession rate of forest dominates the harvesting rate, that is $h<R$. In this case, lower the harvesting rate, higher the forest cover.

There are two steady states of full conservationism. One has no forest cover

$$
A_{3}=(0,1)
$$

and the second one has full forest cover

$$
A_{4}=(1,1)
$$

In the above steady states, the population has the homogeneous opinion of either conservation or non-conservation.

Finally, there are two steady states with mixtures of conservationists and rest non-conservationists. One has no forest cover

$$
A_{5}=\left(0, \frac{\delta-c}{2 \delta}\right)
$$




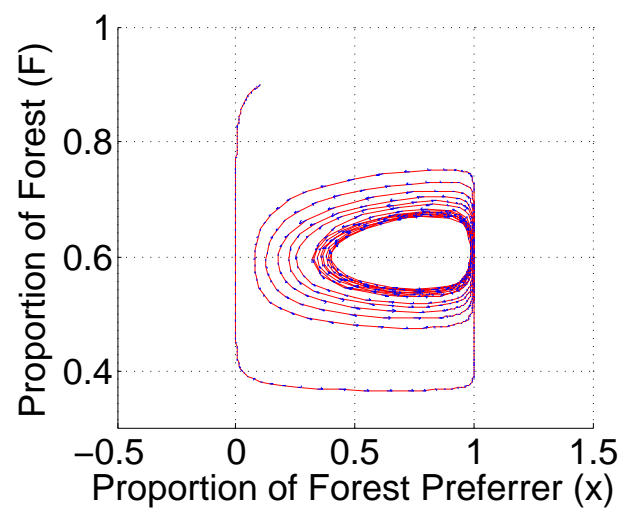

FiguRE 1. Appearance of weakly damped oscillations to an interior equilibrium when the social learning rate $\kappa=2$ /year. All other parameter values are as in Table 1.

whereas the second one has a mix of forest and other land cover

$$
A_{6}=(1+h S, 1+R S)
$$

and this is the only interior equilibrium of the model. Here $A_{5}$ is biologically meaningful when injunctive social norms dominate the conservation value of forest, that is $c<\delta$. $A_{6}$ is biologically meaningful if $0>R S>-1$ and $0>h S>-1$, where $S=\frac{1-c-\delta}{2 R \delta-h}$. Here, $A_{6}$ is biologically meaningful if the absolute value of $S$ is larger than the reciprocal of both $h$ and $R$.

3.1.2. Stability and dynamics. From local stability analysis, $A_{1}=(0,0)$ is locally asymptotically stable (LAS) if the injunctive social norms dominate the conservation value of forest and harvesting dominates the natural succession rate, that is $\frac{\delta}{c}>1$ and $\frac{h}{R}>1$. Here, in the population with a higher harvesting rate, stronger injunctive norms shift the population to the homogeneous opinion so that the state where the entire population are non-conservationists exists. 


$$
A_{2}=\left(1-\frac{h}{R}, 0\right) \text { is LAS if } \frac{h}{R}<1+\delta-c<1 \text { or } \frac{h}{R}<1<1+\delta-c \text {. In either case, }
$$
harvesting is dominated by natural succession. Here, the population can consist entirely of non-conservationists and yet forest can persist at intermediate levels because harvesting is dominated by the rate of natural succession.

$A_{3}=(0,1)$ is always unstable and its instability is reasonable as total forest devastation is impossible in the presence of full forest conservationism, since harvesting always tends to zero as the proportion of conservationists approaches unity.

$A_{4}=(1,1)$ is LAS if injunctive social norms dominate the conservation value of other land use, that is $c+\delta>1$. Here, stronger injunctive social norms force the population to a homogeneous opinion of full conservationism; it does not matter whether harvesting dominates natural succession rate or natural succession dominates harvesting rate.

$A_{5}=\left(0, \frac{\delta-c}{2 \delta}\right)$ is unstable and the instability is reasonable except at $\delta=c$ because total forest devastation is impossible in the presence of conservationism.

From the above results (and neglecting for the moment the interior equilibrium), we conclude that for sufficiently strong injunctive social norms, the population converges either to the state of full non-conservationistm $x=0$ or the state of full conservationism $x=1$. However, in the population where harvesting dominates natural succession with sufficiently strong injunctive social norms, we notice the co-existence of full non-conservationism $A_{1}$ with full-conservationism $A_{4}$. Also, the state of full conservationism $A_{4}$ can coexist with the state of full non-conservationism $A_{2}$.

The interior steady state $A_{6}=(1+h S, 1+R S)$ is LAS if $1+\delta-c<\frac{h}{R}$ or $\frac{2 \delta}{\delta+c}<\frac{h}{R}$, $c+\delta<1,0<2 \kappa \delta\left(1-x^{*}\right) x^{*}<R F^{*}$, where $F^{*}=1+h S$ and $x^{*}=1+R S$. In this case, the injunctive social norms are dominated by the conservation value of other land use. 
Intuitively, the interior equilibrium is stable because injunctive social norms are not strong enough to force the population to either extreme of $x=0$ or $x=1$, and the conservation value of forest is strong enough to support an intermediate level of both forest cover and conservationist opinion, without the support from injunctive social norms. We note that $A_{6}$ cannot coexist with any other steady states.

Local stability analysis does not preclude the possibility of periodic solutions or other nontrivial dynamical behaviour away from steady states. At $\kappa=2 /$ year, numerical simulations suggest the possibility of a stable limit cycle, as conservationism and forest cover appear to oscillate perpetually over very long timescales (Figure 1). The presence of oscillations would be significant: oscillating forest cover is less desirable than stable forest cover since harvesting/restoration cycles could create perpetual disturbances in highly biodiverse climax forest communities. However, we were able to rule out the possibility of a stable limit cycles for certain parameter regimes: for $R>h$ and sufficiently small value of $\kappa \delta$, periodicity in the interior region $D=\{(F, x): 0<F<1,0<x<1\}$ can be ruled out by using Dulac's criterion (Hale and Koçak, 2012, p.373) (for detail see SI Appendix: Appendix A). Thus for $R>h$ and sufficiently small value of $\kappa \delta$, using poincaré-Bendixson theorem (Hale and Koçak, 2012, p.366), the interior equilibrium solution $A_{6}$ is globally asymptotically stable in $D$. Nonetheless, the weakly damped nature of oscillations to the equilibrium interior state are still undesirable due to their implication of harvesting/restoration cycles in the population and the ubiquitous presence of noise, which can sustained weakly damped oscillations indefinitely. We also note that stable limit cycles can occur at other parameter values (see below). 

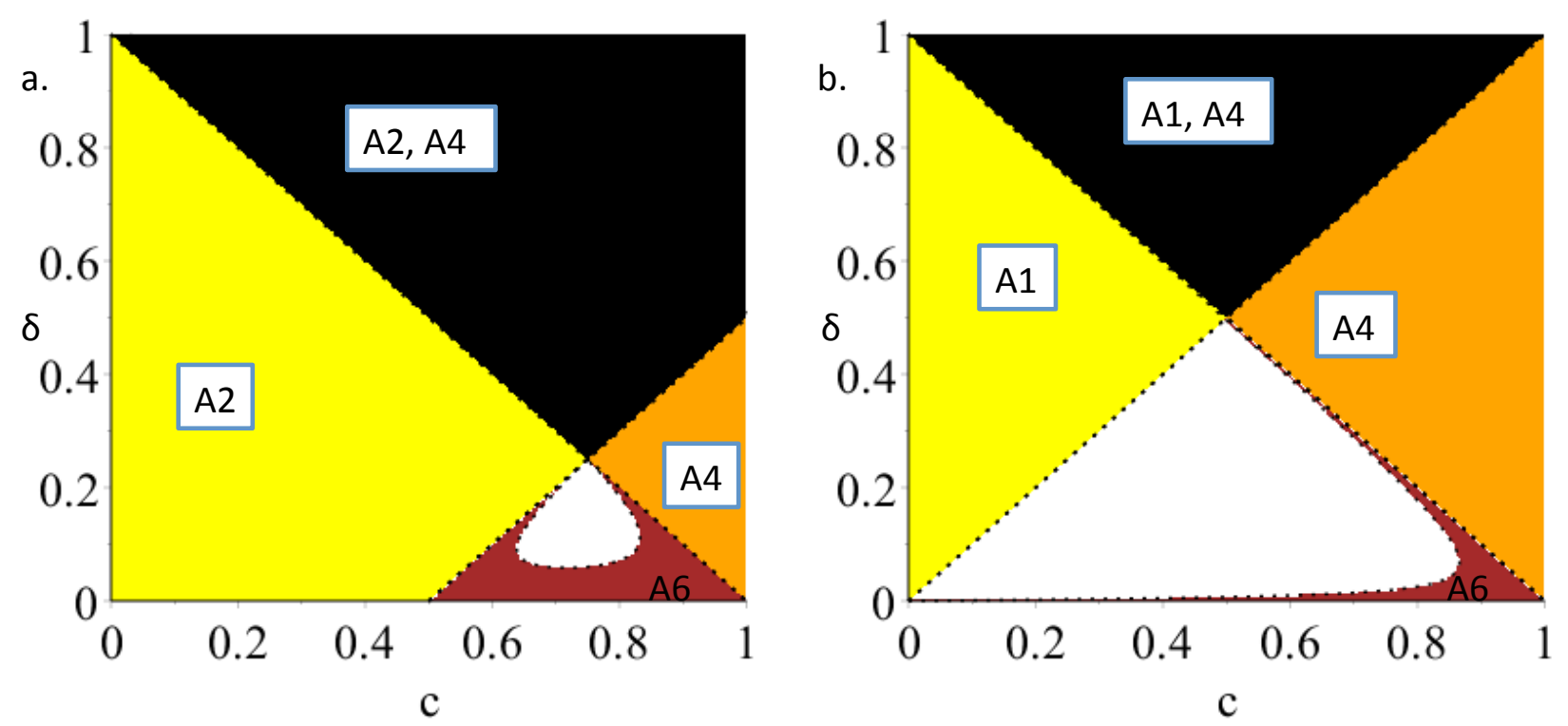

FIGURE 2. The relationship between harvesting rate and natural succession rate influences the region where all equilibria are unstable. Subpanels show (a) succession dominating harvesting $h=0.01 /$ year, $R=0.02 /$ year and (b) harvesting dominating succession $h=0.02$ /year, $R=0.01 /$ year. The equilibria in the subpanels are stable in the corresponding region. White is the region of instability for all steady states and the presence of stable limit cycles. All other parameters except those being varied appear in Table 1.

Parameter planes provide significant insight into the conditions of stability of the various steady states. From the $c-\delta$ parameter plane, we observe major changes in the stability paradigm when we reverse the relative magnitude of the harvesting rate and the natural succession rate. Stability of a state of mixed land with full non-conservationism $A_{2}$ (Figure 2a) has nearly been replaced by the stability of a state of no-forest cover with full nonconservationism $A_{1}$ (Figure $2 \mathrm{~b}$ ). Also the region of stability of interior steady state has been drastically decreased, resulting in a larger region of instability for all steady states. In this region of instability, numerical simulations indicate the presence of stable limit cycles.

In bifurcation diagrams we observe that even a small harvesting rate can cause the oscillations in the system. Decreasing the social learning rate $\kappa$ stabilizes the system by reducing 

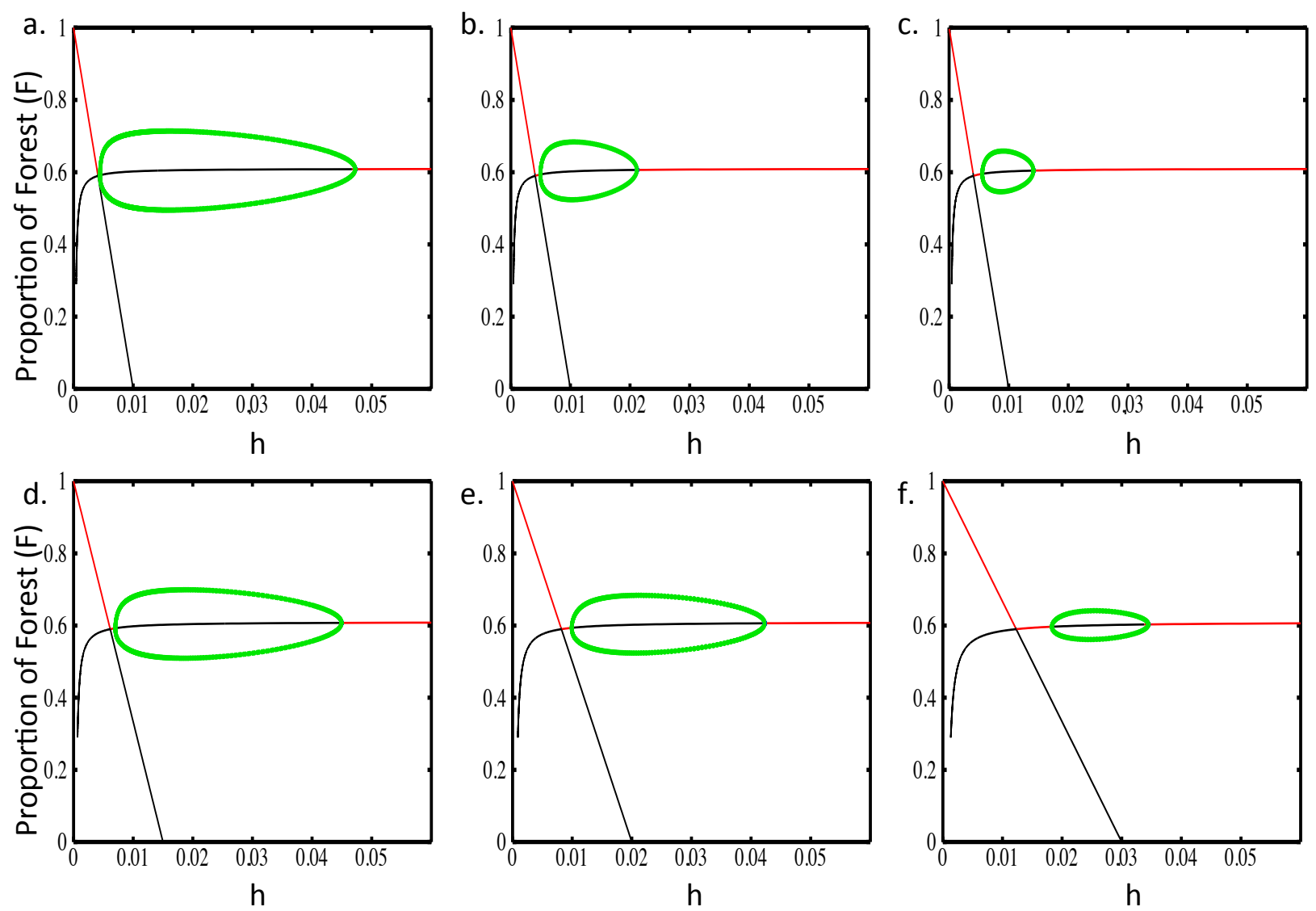

FiguRE 3. Decreasing the social learning rate $k$ or increasing the natural succession rate $R$ helps reduce the tendency to oscillate. Subpanels show bifurcation diagrams of forest cover $F$ versus harvesting $h$ for various values of $\kappa$, (a) 4/year (b) 2/year (c) 1.5/year, and various values of $R$ (d) $0.015 /$ year (e) $0.02 /$ year (f) $0.03 /$ year. $\kappa=4$ /year in subpanels (d-f); all other parameters except those being varied appear in Table 1 .

the range of parameters $h$ for which oscillatory solutions are obtained (Figure 3a-c). Similarly, increasing the natural succession rate $R$ (Figure 3d-f), reduces the parameter range for which oscillations occur. Also the oscillations that appeared due to small conservation value can be reduced and removed with the increase in the conservation value (SI Appendix Fig.S2 a-c). But an increase in injunctive social norms increases oscillations for a larger range of harvesting (SI Appendix Fig.S2 d-f). 
The time evolution of model dynamics helps illustrate the role of injunctive social norms in the model. For certain initial conditions, injunctive social norms do not favour conservationism. When $x$ is initially small, human preference starts increasing during an early phase of forest destruction (Figure 4a). For higher values of injunctive social norms, it may take several years to centuries to manifest change in human preference and thus in forest cover (Figure 4b, c). Also based on our baseline parameter values, society is practicing a higher harvesting rate than the natural succession rate of forest. As a result, stronger social norms support harvesting and lead the forest to decline. Thus stronger social norms gradually increase the population opinion homogeneity, eventually dominating conservation priorities for the rare and endangered species. The situation is different over a larger time window, in which an increase in injunctive social norms compel the forest cover to accelerate in both direction as well as helps to reduce the oscillation (SI Appendix Fig.S1). Note that the situation will be different when we change the initial conditions such that $x$ is initially higher: in these cases, social norms will move population to greater conservationism (SI Appendix Fig.S3 a-c).

Because of conservation priorities to protect forest ecosystems, forest cover can be maintained at an optimum level even when only $20 \%$ of forest cover remains initially (Figure $4 \mathrm{~d}$ ). Also if there is a larger difference between initial prevalence of conservationism and initial forest cover, forest cover will initially decline before it starts to recover (Figure 4e,f). 

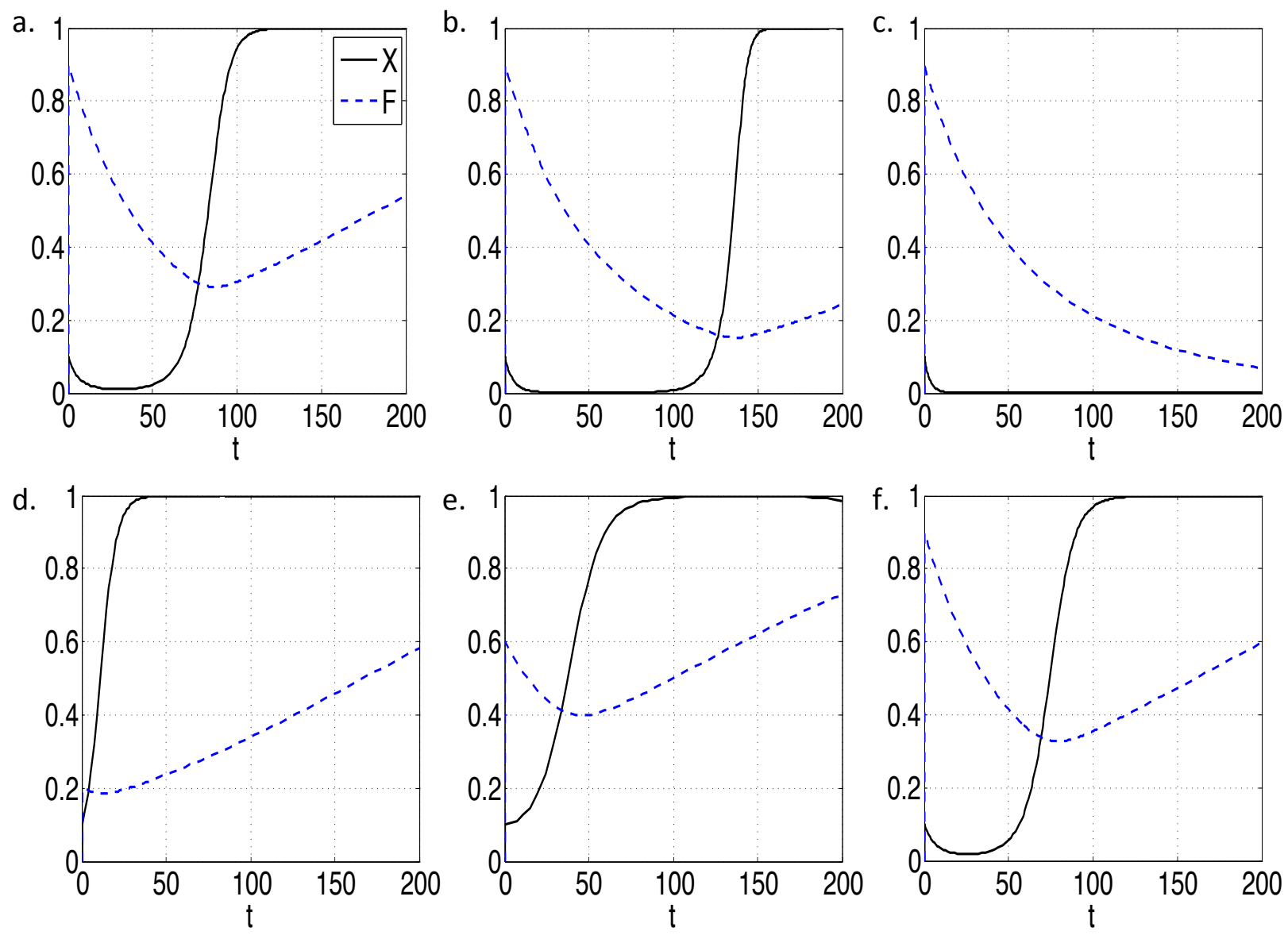

FiguRE 4. For the initial condition $\left(F_{0}, x_{0}\right)=(0.9,0.1)$, increasing the strength of injunctive social norms $\delta$, (a) 0.05 (b) 0.2 (c) 0.4 , decrease conservationism and thus forest cover. Varying the initial value of forest cover $F_{0}$, (d) 0.2 (e) 0.6 (f) 0.9 does not affect much the forest cover in longer time window. All other parameters except those being varied appear in Table 1.

\subsection{Model without injunctive social norms.}

3.2.1. Model equilibria. Substituting $\delta=0$ in equation (4), the coupled HES model reduces to a model with the following system of equations:

$$
\begin{aligned}
& \dot{x}=\kappa x(1-x)(c-F) \\
& \dot{F}=R F(1-F)-h(1-x) F .
\end{aligned}
$$


From equation (5), there are two steady states $\left(F^{*}, x^{*}\right)$ of full non-conservationism of forest. One has no forest cover

$$
B_{1}=(0,0)
$$

and the second has a mix of forest and other land cover

$$
B_{2}=\left(1-\frac{h}{R}, 0\right) .
$$

$B_{2}$ is biologically meaningful when natural succession rate dominates the harvesting rate, that is, $\frac{h}{R}<1$. In this case, a lower harvesting rate results in higher forest cover.

There are two steady states of full conservationism. One has no forest cover

$$
B_{3}=(0,1)
$$

and the second one has full forest cover

$$
B_{4}=(1,1)
$$

In the above steady states, the population has the homogeneous opinion of either full conservationism or full non-conservationism.

Note that steady states $B_{1}, B_{2}, B_{3}, B_{4}$ are the same as steady states $A_{1}, A_{2}, A_{3}, A_{4}$ of the model with injunctive social norms, and $A_{5}$ of the social norms model is no longer meaningful in this case.

The interior equilibrium $A_{6}$ of the model with social norms reduces to:

$$
B_{5}=\left(c, 1-\frac{R}{h}(1-c)\right)
$$


and it is biologically meaningful when the ratio of the harvesting rate to the succession rate dominates the relative conservation value of other land cover, that is $\frac{h}{R}>1-c$. Here, larger conservation values result in higher forest cover and prevalence of conservationism.

3.2.2. Stability and dynamics. The absence of injunctive social norms has a large impact on the stability of the coupled HES. The steady states of full non-conservationism and no forest cover, and full conservationism and full forest cover, can no longer be stable, making conservation priorities based on the rarity of forest more important for dynamics.

$B_{1}=(0,0)$ is unstable and its instability in the absence of injunctive social norms is reasonable as extreme rarity of forest should cause $x$ to diverge away from zero, causing $F$ to rise as well. This contrasts with the finding that $A_{1}=(0,0)$ can be stable for certain parameter regimes in the model with injunctive norms.

$B_{2}=\left(1-\frac{h}{R}, 0\right)$ is LAS if the ratio of harvesting rate to natural succession rate is less than the relative conservation value of other land, that is $\frac{h}{R}<1-c . A_{2}$ can no longer exist if a conservationist exists in the population. $x=0$ implies $\dot{x}=0$ and thus the line $x=0$ is invariant. Thus, the only one locally asymptotically stable staedy state $B_{2}$ at the boundary is globally asymptotically stable (GAS) at $x=0$.

$B_{3}=(0,1)$ is unstable and its instability is reasonable because forest cover cannot remain zero in the presence of full conservationism. $B_{4}=(1,1)$ is also unstable because full conservationism cannot remain stable in the presence of full forest cover, due to competing needs for other uses of land. This contrasts with the finding that $A_{4}$ can be stable for certain parameter regimes in the model with social norms.

The interior equilibrium $B_{5}=\left(c, 1-\frac{R}{h}(1-c)\right)$ is LAS if the ratio of the harvesting rate to the natural succession rate is greater than the relative value of other land uses, that is 
$\frac{h}{R}>1-c . \quad B_{5}$ is LAS if it is biologically meaningful. Periodicity in the interior region $D=\{(F, x): 0<F<1,0<x<1\}$ can be ruled out without any parameter restriction by using Dulac's criterion (Hale and Koçak, 2012, p.373) (for detail see SI Appendix: Appendix B). Thus using poincaré-Bendixson theorem (Hale and Koçak, 2012, p.366), the interior equilibrium $B_{5}$ is GAS in $D$ when it exists. Note that the stability analysis of above five steady states is conducted in detail in SI Appendix: Appendix B.

In the $h-R$ parameter plane (Figure 5a-c), we observe significant differences from the model with social norms included. We only observe two stable equilibria, $A_{2}$ and $A_{5}$. Increasing the conservation value of forest shrinks the region of stability of pure non-conservationism $B_{2}$ and thus increases the region of stability for the interior steady state $B_{5}$. Also, as the forest cover in the interior steady state directly depends upon the conservation value $c$, forest cover increases with the increase in $c$.
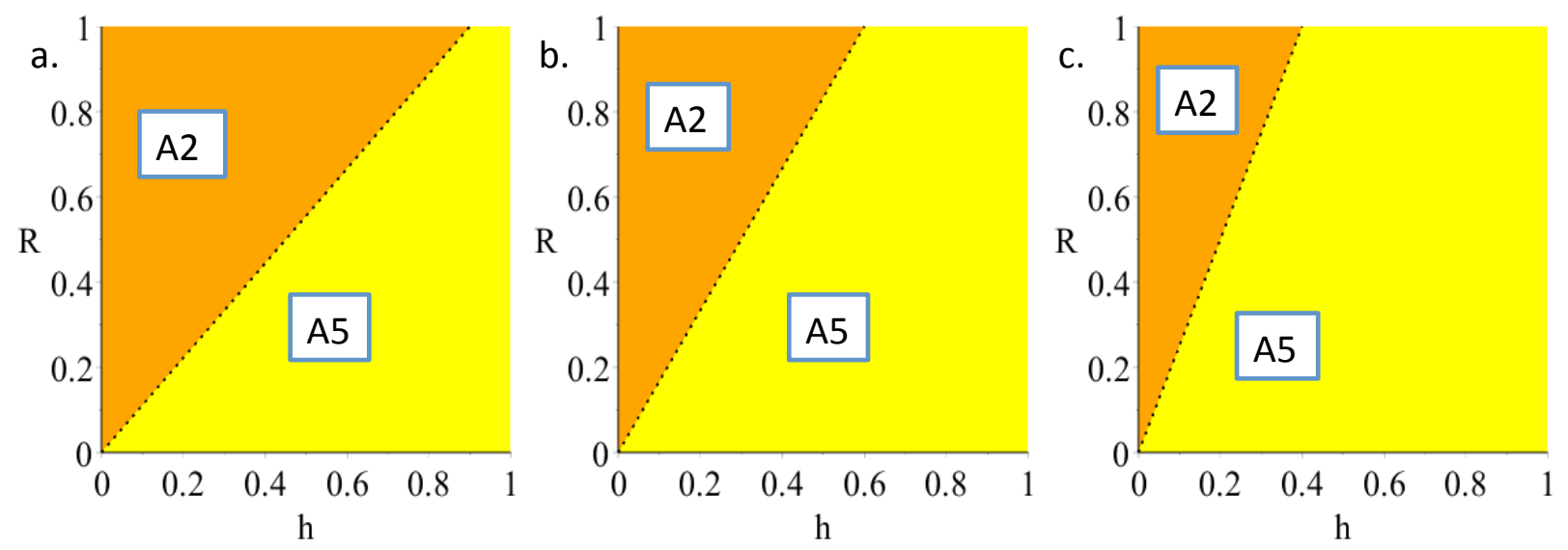

FiguRE 5. An increase in conservation value $c$ increases the region of stability of the interior equilibrium $A_{5}$ and thus decreases the stability region for the boundary equilibrium $A_{2}$. For (a) $c=0.1$ (b) $c=0.4$ (c) $c=0.6, A_{5}$ and $A_{2}$ are stable in the labeled regions. All other parameters except those being varied appear in Table 1. 

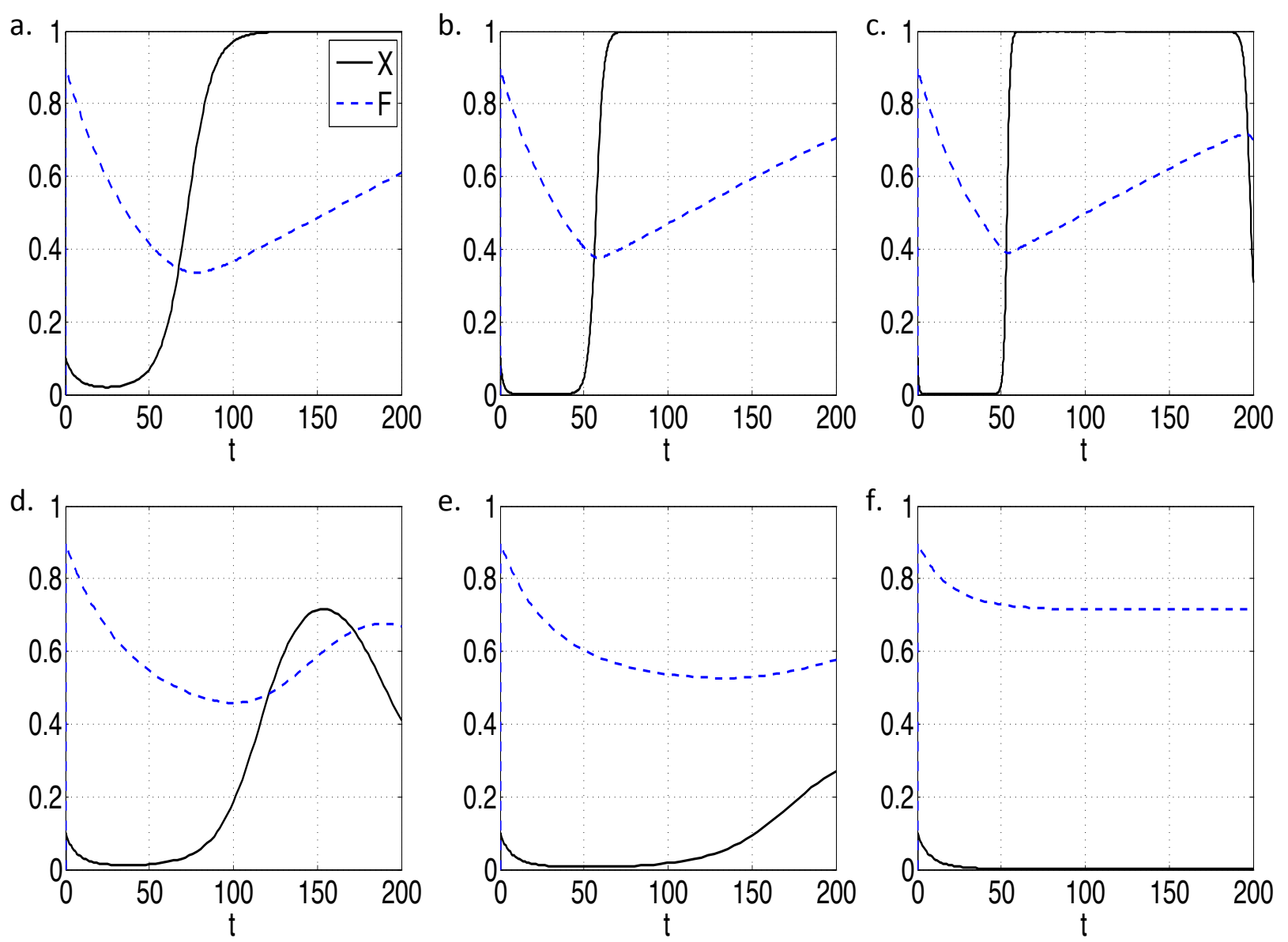

FIGURE 6 . Increasing the social learning rate $\kappa$ results in more rapid turnover, while increasing the natural succession rate $R$ increases forest cover but not conservationism. Impact of variation in social learning rate $\kappa /$ year, (a) 0.5 (b) 2 (c) 5 and natural succession rate $R /$ year, (d) 0.03 (e) 0.04 (f) 0.07 ; all other parameters except those being varied appear in the table 1.

A rapid increase in the social learning rate has both positive as well as negative impacts on the system. A low social learning rate results in slow decline of forest but also a slow recovery rate (Figure 6a), whereas a higher social learning rate increases both the rate of forest destruction as well as the rate of forest recovery, at least up unto its natural maximum $R$ (Figure 6b, c). A higher social learning rate also results in rapid transitions between population states and decreases the period of damped oscillations. If the natural succession rate of forest is increased, forest cover dominates other land cover most of the time (Figure 
6d-f). Also, a higher succession rate guarantees sustainable forest cover for a longer time window. Conservationism declines on account of the abundance of forest, but because $R$ is high, forest cover can be maintained even in the absence of public support.
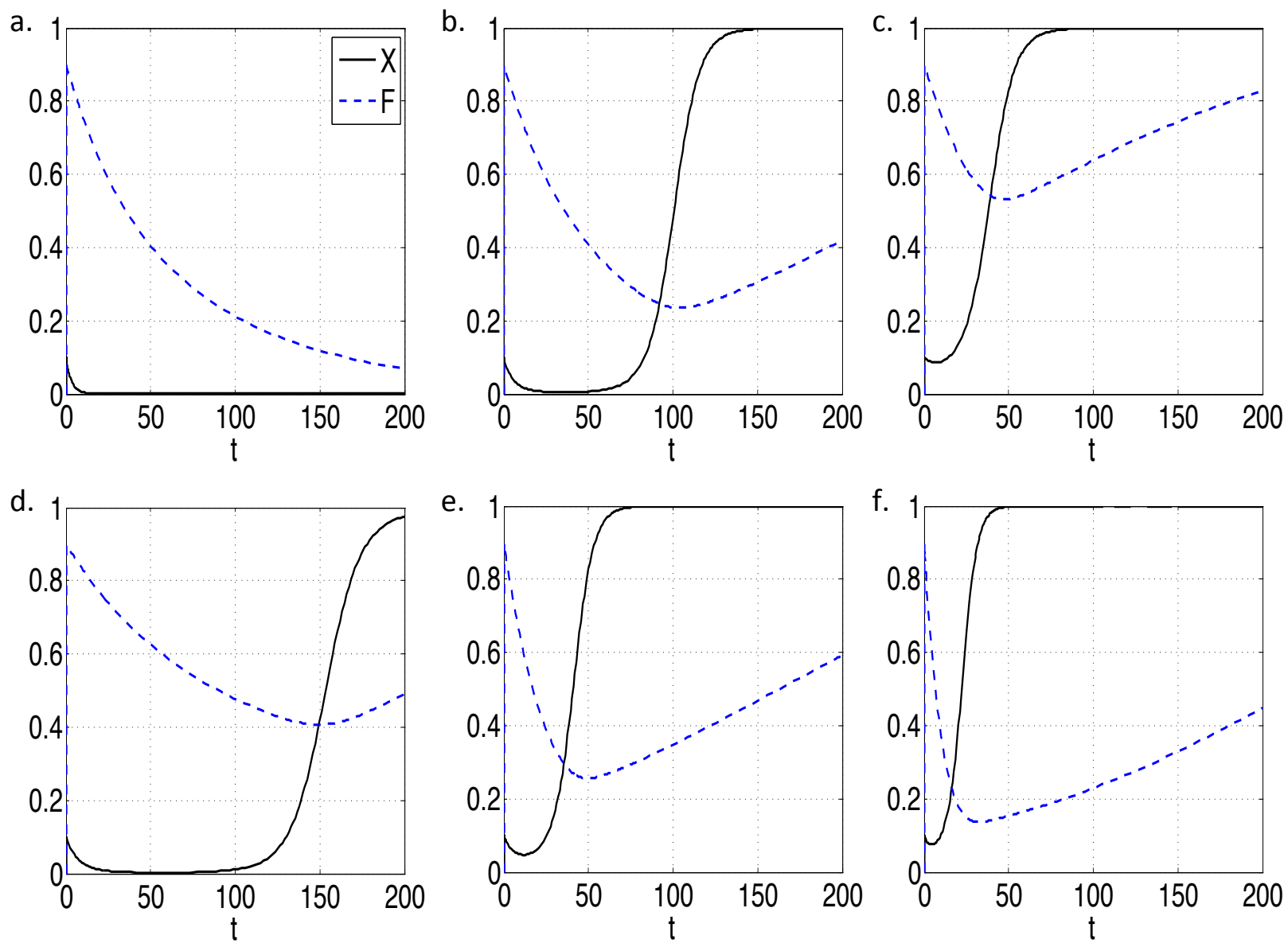

FigURE 7. Increasing the conservation value of forest $c$ supports forest cover and conservationism, while increasing the harvesting efficiency $h$ reduces forest cover but also stimulates conservationism and thus forest recovery. Impact of variation in relative conservation value of forest $c$, (a) 0.2 (b) 0.5 (c) 0.8 and harvesting rate $h /$ year, (d) 0.01 (e) 0.04 (f) 0.1 ; all other parameters except those being varied appear in the table 1.

A higher conservation value of forest $c$ helps to increase conservationism and thus forest cover (Figure 7a-c). It also stops the sharp increase in forest destruction by changing the human preference rapidly. A higher harvesting rate increases the rate of forest decline, but it also stimulates a more rapid response from conservationism, causing a decline in the 
effective harvesting rate $h(1-x)$ (Figure $7 \mathrm{~d}-\mathrm{f}$ ). As a result, when $h$ is large enough, full conservationism is stable and the forest can recover at its maximal rate $R$.

\section{Discussion}

Here we compared dynamics of a coupled HES model for forest growth and conservationist opinion in a human population when injunctive social norms were included to when injunctive social norms were excluded. We found that the model with injunctive social norms had five possible obserable outcomes (four stable equilibrium, plus stable limit cycles) whereas the model without social norms only had two stable equilibria. In the case of the model with injunctive social norms, stable equilibria could also co-exist, while in the case of the model without social norms, the two stable equilibria are simply globally asymptotically stable whenever they exist in their respective parts of parameter space. The complexity in the case of the model with social norms arises because injunctive social norms drive dynamics to the boundaries of the phase space, while rarity-based conservation drives it back to the interior. These results were established with a mixture of numerical and qualitative analysis, including establishment of global asymtotic stability using the Dulac criterion and other methods.

The model yielded other interesting predictions. In general, if human opinion is based only on the desire to protect endangered ecosystems, destruction of the whole forest as well as the existence of full forest cover should not be possible. However, injunctive social norms make it possible because when the norms are universally accepted, the population persists at the boundary of phase space for a long period of time, even the punishment efforts has already been stopped (Helbing et al., 2010). At this point the conservation value of rare ecosystems is overshadowed by social psychology. 
For the model with injunctive social norms, the local and the global stability of the interior steady state are tied to complex parameter restriction, whereas the situation is simple when social norms are absent. As an example, the periodicity at the interior steady state of the model with social norms can be ruled out under certain parameter restriction but it can be easily ruled out without any condition when social norms are absent.

Our analysis also shows that without other supports, even the nominal rate of harvesting ( $2 \%$ as our baseline value, which is small compared to the rate under current practice in many low-income countries) can lead to removal of almost all forest cover. In a coupled HES setting, a population may eventually respond by demanding conservationism when forest cover becomes to low, leading to a partial counteracting of higher maximal harvesting rates. However, this mechanism does not work perfectly and only conserves forest at the cusp of extinction. In contrast, increasing the conservation value of forest $(c)$ has the uniform effect of both boosting and stabilizing forest cover as well as supporting conservationism in the population. Thus we suggest policy that teaches the conservation value of forests can be an effective way to help preserve forests and the species that depend on them.

Our model made simplifying assumptions in the name of analytical tractability, some of which might influence our predictions. For instance, we ignored stochastic effects, which could be important in modelling the continued persistence or extinction of ecosystems at risk. We also assumed a single, closed population, whereas in fact both human populations and forest systems are open and connected to other populations from which species (or opinions) can be rescued when endangered in any one patch. Similarly, we did not include other aspects of real systems such as population or spatial heterogeneities. These simplifying assumptions can and should be relaxed in future research so that mathematical models of 
coupled human-environment system dynamics can provide more insights and better inform environmental policy.

\section{REFERENCES}

An, L., Linderman, M., Qi, J., Shortridge, A., and Liu, J. (2005). Exploring complexity in a human-environment system: an agent-based spatial model for multidisciplinary and multiscale integration. Annals of the association of American geographers, 95(1):54-79.

Anand, M., Laurence, S., and Rayfield, B. (2005). Diversity relationships among taxonomic groups in recovering and restored forests. Conservation Biology, 19(3):955-962.

Barlow, L.-A., Cecile, J., Bauch, C. T., and Anand, M. (2014). Modelling interactions between forest pest invasions and human decisions regarding firewood transport restrictions. PloS one, 9(4):e90511.

Bauch, C. T., Sigdel, R., Pharaon, J., and Anand, M. (2016). Early warning signals of regime shifts in coupled human-environment systems. Proceedings of the National Academy of Sciences of the USA, in press.

Davis, A. et al. (2001). A forestry program for oregon: Oregonians discuss their opinions on forest management \& sustainability. A Quantitative Research Project. Davis, Hibbits and McCaig Inc. Portland, Oregon.

Fearnside, P. M. (1993). Deforestation in brazilian amazonia: the effect of population and land tenure. Ambio-Journal of Human Environment Research and Management, $22(8): 537-545$.

Francis, R. A. and Krishnamurthy, K. (2014). Human conflict and ecosystem services: finding the environmental price of warfare. International Affairs, 90(4):853-869. 
Hale, J. K. and Koçak, H. (2012). Dynamics and bifurcations, volume 3. Springer Science \& Business Media.

Heinen, J. T. and Yonzon, P. B. (1994). A review of conservation issues and programs in nepal: from a single species focus toward biodiversity protection. Mountain Research and Development, pages $61-76$.

Helbing, D., Yu, W., Opp, K.-D., and Rauhut, H. (2010). The emergence of homogeneous norms in heterogeneous populations. American Journal of Sociology, submitted.

Henderson, K., Bauch, C. T., and Anand, M. (2016). Alternative stable states and the sustainability of forests, grasslands and agriculture. Proceedings of the National Academy of Sciences of the USA, in press.

Henderson, K. A., Anand, M., and Bauch, C. T. (2013). Carrot or stick? modelling how landowner behavioural responses can cause incentive-based forest governance to backfire. PloS one, 8(10):e77735.

Innes, C., Anand, M., and Bauch, C. T. (2013). The impact of human-environment interactions on the stability of forest-grassland mosaic ecosystems. Scientific reports, 3 .

Lade, S. J., Tavoni, A., Levin, S. A., and Schlüter, M. (2013). Regime shifts in a socialecological system. Theoretical ecology, 6(3):359-372.

Liu, J., Dietz, T., Carpenter, S. R., Folke, C., Alberti, M., Redman, C. L., Schneider, S. H., Ostrom, E., Pell, A. N., Lubchenco, J., et al. (2007). Coupled human and natural systems. AMBIO: a journal of the human environment, 36(8):639-649.

Marten, G. G. (2001). Human ecology: Basic concepts for sustainable development. Earthscan. 
Masek, J. G., Cohen, W. B., Leckie, D., Wulder, M. A., Vargas, R., de Jong, B., Healey, S., Law, B., Birdsey, R., Houghton, R., et al. (2011). Recent rates of forest harvest and conversion in north america. Journal of Geophysical Research: Biogeosciences, 116(G4).

McCracken, K. (2013). Sudbury: The journey from moonscape to sustainably green. ActiveHistory.ca, History Matters. http://activehistory.ca.

Millennium Ecosystem Assessment, . (2005). Ecosystems and human well-being. Washington, $D C$.

Mogaka, H. (2001). Economic aspects of community involvement in sustainable forest management in Eastern and Southern Africa. Number 8. IUCN.

Oraby, T., Thampi, V., and Bauch, C. T. (2014). The influence of social norms on the dynamics of vaccinating behaviour for paediatric infectious diseases. Proceedings of the Royal Society of London B: Biological Sciences, 281(1780):20133172.

Overbeck, G. E., Müller, S. C., Fidelis, A., Pfadenhauer, J., Pillar, V. D., Blanco, C. C., Boldrini, I. I., Both, R., and Forneck, E. D. (2007). Brazil's neglected biome: the south brazilian campos. Perspectives in Plant Ecology, Evolution and Systematics, 9(2):101-116. Rockström, J., Steffen, W., Noone, K., Persson, Å., Chapin, F. S., Lambin, E. F., Lenton, T. M., Scheffer, M., Folke, C., Schellnhuber, H. J., et al. (2009). A safe operating space for humanity. Nature, 461(7263):472-475.

Rodrigues, A., Koeppl, H., Ohtsuki, H., and Satake, A. (2009). A game theoretical model of deforestation in human-environment relationships. Journal of theoretical biology, 258(1):127-134.

Satake, A. and Iwasa, Y. (2006). Coupled ecological and social dynamics in a forested landscape: the deviation of individual decisions from the social optimum. Ecological Research, 


\section{$21(3): 370-379$.}

Satake, A., Janssen, M. A., Levin, S. A., and Iwasa, Y. (2007). Synchronized deforestation induced by social learning under uncertainty of forest-use value. Ecological Economics, 63(2):452-462.

Scholes, R. and Archer, S. (1997). Tree-grass interactions in savannas. Annual review of Ecology and Systematics, pages 517-544.

WWF (2013). World Wildlife Fund. www.worldwildlife.org/threats/deforestation, www.worldwildlife.org/habitats/forest-habitat. 